\title{
SURFACE PHOTOMETRY OF LOW-SURFACE-BRIGHTNESS GALAXIES IN THE AREA OF THE NGC 972 GROUP OF GALAXIES
}

\author{
J. VENNIK ${ }^{1}$ and G.M. RICHTER ${ }^{2}$ \\ ${ }^{1}$ Tartu Astrophysical Observatory, EE2444 Toravere, Estonia \\ ${ }^{2}$ Astrophysical Institute Potsdam, An der Sternwarte 16, 14482 Potsdam, Germany
}

\begin{abstract}
A nearby group of galaxies, centred on NGC 972 and conspicuously rich in faint dwarf spheroidal galaxies, has been investigated photometrically on $B$ and $V$ plates obtained with the Tautenburg $2 \mathrm{~m}$ Schmidt telescope. For six low surface brightness galaxies, the equivalent B- and V-brightness profiles have been extracted, and asymptotic magnitudes and mean colours have been estimated. Their equivalent profiles are well fitted by modified isothermal (King) models.
\end{abstract}

There is a nearby loose group of 7 spiral and irregular galaxies - NGC 972, NGC 1012, NGC 1056, UGC 1958, UGC 2017, UGC 2053, UGC 2221 - with similar redshifts, centred on the luminous galaxy NGC 972, in the foreground of the rich Pisces-Perseus supercluster. In the group area there is a conspicuous concentration of four extremely faint dwarf spheroidals, detected on POSS copies and catalogued by Karachentseva (1968) - K 16, K 17, K 19 and K 21. Despite the lack of measured redshifts for these dwarf spheroidals, we have good reason to assume them to be related to the bright parent galaxy NGC 972 because:

1) it is generally accepted that dwarf spheroidals tend to be concentrated in rich clusters of galaxies or are related to luminous parent galaxies in the field;

2) the background Pisces-Perseus supercluster is too far away for the detection of such faint cluster members.

In the present paper we study the brightness distribution in the low-surface-brightness (hereafter LSB) dwarf members of the NGC 972 group.

The $B$ and V photographic plates were obtained with the $2 \mathrm{~m}$ Schmidt telescope at the Tautenburg Observatory. The digitized images of galaxies were processed in the MIDAS environment, applying the photometric software package developed at the Astrophysical Institute Potsdam. The raw images were cleaned by means of the adaptive filtering technique, described by Richter (1978). Magnitude zero-point calibration was performed on the basis of the photoelectric aperture photometry of NGC 972, taken from Longo et al. (1983). The equivalent brightness profiles were subsequently extracted from the cleaned images and asymptotic total magnitudes determined.

In Fig. 1 the B- and V-profiles for four dwarf spheroidals are presented. Surface brightnesses (SB) are plotted over the equivalent radius $\left(\mathbf{R}_{e q}\right)$, which is defined as the radius of the circle of the same area as that inside the given isophote. 


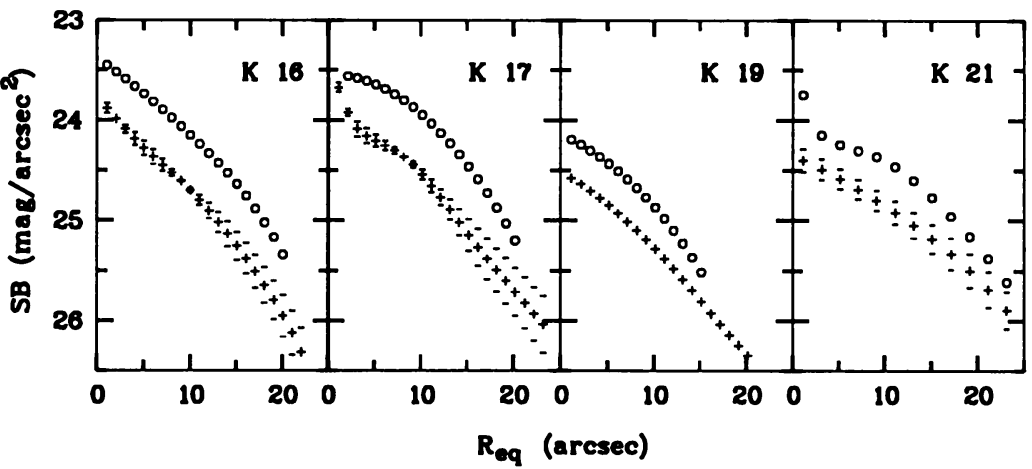

Figure 1. Equivalent surface brightness (SB) profiles of four dwarf spheroidals in the NGC 972 group area. Open circles denote the V-profile; crosses the mean B-profile with error bars, determined from measurements on two plates.

Table 1 lists the following photometric parameters of six LSB galaxies in the NGC 972 group: 1) galaxy identification; 2) $R_{25.5}$ - equivalent radius (in arcseconds) at the $25.5 \mathrm{~B} / \mathrm{arcsec}^{2}$ isophote; 3) $R_{e}$ - effective radius in arcseconds; 4) $\mu_{e}$ - mean surface brightness inside $R_{e}$ in $\mathrm{B} / \operatorname{arcsec}^{2}$; 5) $\mathrm{B}_{25.5}$ - total B-magnitude inside the $25.5 \mathrm{~B} / \operatorname{arcsec}^{2}$ isophote; 6) $\mathrm{B}_{T}$ - asymptotic B-magnitude; 7) B-V - mean colour index; 8) $\mathbf{M}_{B}$ - absolute B-magnitude, calculated for distance modulus 31.7. Columns $9-11$ present the King model parameters: $r_{t} / r_{c}$ - concentration index; $r_{c}$ - core radius in arcseconds and $\mu_{c}-$ central SB in $B / \operatorname{arcsec}^{2}$.

Table 1.

\begin{tabular}{|c|c|c|c|c|c|c|c|c|c|c|}
\hline Galaxy & $\mathbf{R}_{2 s s}$ & $\mathbf{R}$. & $\mu_{.}$ & $\mathbf{B}_{2 s s}$ & $\mathbf{B}_{\boldsymbol{T}}$ & B.V & $\mathbf{M}_{\boldsymbol{B}}$ & $\mathbf{r}_{\boldsymbol{t}} / \mathbf{r}_{\mathrm{c}}$ & $\mathbf{r}_{\mathbf{e}}$ & $\boldsymbol{\mu}_{c}$ \\
\hline UGC 2017 & 26 & 16.5 & 24.2 & 16.4 & 16.1 & 0.41 & -15.6 & 1.00 & 6.0 & 23.58 \\
\hline UGC 2053 & 35 & 20 & 23.5 & 15.2 & 15.0 & 0.30 & -16.6 & 0.50 & 15.1 & 23.06 \\
\hline K 16 & 17 & 12 & 24.5 & 17.3 & 17.1 & 0.56 & -14.6 & 0.50 & 9.8 & 24.02 \\
\hline K 17 & 18 & 13.7 & 24.5 & 17.2 & 16.8: & 0.54 & -14.9 & 0.50 & 11.2 & 24.04 \\
\hline K 19 & 12 & 14.5 & 25.2 & 18.4 & 17.4: & 0.44 & -14.3 & 0.75 & 7.2 & 24.59 \\
\hline K 21 & 19 & 15.8 & 24.9 & 17.4 & 16.9: & 0.41 & -14.8 & 0.50 & 14.1 & 24.48 \\
\hline
\end{tabular}

According to Binggeli \& Cameron (1991), faint dwarf galaxies $\left(M_{B} \geq-16\right)$ are mostly spheroidals and can be fitted by the King model. On the other hand, Impey et al. (1988) argue that, according to reliable CCD data, the radial SB-profiles of low luminosity galaxies are mostly exponential. In Fig. 1 the logarithmic surface brightness is plotted over linear equivalent radius. The exponential brightness profiles should be straight lines in this coordinate system. As seen from Fig. 1 the SB-profiles of faint spheroidals clearly deviate from the exponential distribution. According to the classification of Binggeli \& Cameron (1991) the 'subexponential' SB-profiles of our dwarfs belong to class $\mathrm{V}$ - with central brightness deficient. This profile type should be characteristic for dwarf ellipticals. K 17 and possibly K 16 seem to have blue stellar nuclei, partially smeared out due to poor seeing and poor signal to noise ratio. 
If the four faint dwarf spheroidals belong to the NGC 972 group, their asymptotic magnitudes yield absolute B magnitudes between -14.3 and -14.9 . Their relatively blue B-V colour indices between 0.41 and 0.56 are poorly determined because of the poor signal to noise ratio of the $V$ plates used. Modified isothermal models (King models) provide good fits to observed SBprofiles.

If the NGC 972 group is a real bound system of physically related galaxies, a large amount of dark matter is needed to meet the large redshift residuals of its members. The density distribution in low mass dwarf satellites is, in this case, strongly influenced by the massive dark halo of the group they are moving in.

\section{References}

Binggeli, B. and Cameron, L.M., 1991. Astron. Astrophys., 252, 27.

Impey, C., Bothun, G. and Malin, D., 1988. Astrophys. J., 330, 634.

Karachentseva, V.E., 1968. Soob. Byur. Obs., 39, 61.

King, I., 1966. Astron. J., 71, 64.

Longo, G. and de Vaucouleurs, A., 1983. 'A general catalogue of photoelectric magnitudes and colors in the UBV system of 3578 galaxies brighter than the 16-th V-magnitude (1936-1982), Univ. of Texas, Austin.

Richter, G.M., 1978. Astron. Nachr., 299, 283. 